\title{
Measuring Radar Signatures of a Simple Pendulum using Cantenna Radar
}

\author{
Archit Harsh \\ Masters Student, Mississippi State University, Starkville, USA, 39759 \\ ah2478@msstate.edu
}

\begin{abstract}
This paper gives a detailed analysis of the physics of simple pendulum and the equations governing the motion and velocity. The pendulum works in three modes: simple, damped and driving and driving only. The signatures are evaluated and simulated by the means of four different approaches: Euler method, Euler-Cromer method, $2^{\text {nd }}$ order Runge-kutta method and built-in ODE-23 matlab solver. The simulation results are compared to the measured radar signatures using a CANTENNA RADAR originally developed by MIT. The radar was operated in Doppler mode and the micro-Doppler effects associated with pendulum is studied. This paper attempts to provide an in-depth background and analysis of how the pendulum works and the associated micro-Doppler study using RADAR.
\end{abstract}

\section{Keywords}

Micro-Doppler; Radar; Matlab; Pendulum

\section{Academic Discipline And Sub-Disciplines}

Computer and Technology, Signal Processing, Electrical Engineering

\section{TYPE (METHOD/APPROACH)}

Computational Simulations, Real time experiments

\section{INTRODUCTION}

A pendulum is a weight suspended from a pivot so that it can move freely[1]. When a weight is displaced sideways from its resting equilibrium position, it is subject to a restoring force due to gravity that will accelerate it back towards the equilibrium position. When released, the restoring force combined with the pendulum's mass causes it to oscillate about the equilibrium position, swinging back and forth. The time for one complete cycle, a left swing and a right swing, is called the period. The simple pendulum is an idealized mathematical model of a pendulum. When given a push, it will swing back and forth at constant amplitude. This model does not account for friction and hence not practical. Real pendulum or damped pendulums are subject to friction and air-drag, so the amplitude of their swing declines[1]. This paper talks about the physics of both these types of pendulums and studies the doppler signatures associated with it. The results are simulated in MATLAB using four different approaches. The simulated results are then compared to the experimental results carried out by a self-designed CANTENNA RADAR for validating the Doppler-effect associated with the pendulum.

This paper is organized as follows: Sections 1.1 and 1.2 overviews equations governing the motion of pendulum and comparison of four different approaches used in this paper. Section 2 presents simulation results for modelling the simple and physical pendulums. The results are evaluated for calculating the angle and displacement w.r.t time using four different approaches. Section 3 provides the experimental setup using the CANTENNA RADAR and underlines the experimental parameters used in the study. Finally, the experimental results are presented for validating the theoretical results.

\section{Equations governing motion of a pendulum:}

The differential equation governing the motion of a simple pendulum is given by:

$$
\frac{d^{2} \theta}{d l^{2}}+\frac{g}{l} \sin \theta=0
$$

Where, $\mathrm{g}$ is acceleration due to gravity, $\mathrm{I}$ is the length of the pendulum, and $\theta$ is the angular displacement.

Given the initial conditions $\theta(0)=\theta_{0}$ and $\frac{d \theta}{d t(0)}=0$, the solution becomes,

$$
\theta(t)=\theta_{0} \cos \left(\sqrt{\frac{g}{l}} t\right) ; \quad \theta_{0}<<1
$$


This motion is simple harmonic motion where $\theta$ is semi-amplitude of the oscillation. The period of motion is, then given by:

$$
T=2 \pi \sqrt{\frac{l}{g}} ; \theta_{0}<<1
$$

Which is commonly known as Christian Huygen's law for the period. Note that under small angle approximations, the period is independent of the amplitude $\theta_{0}$.

From these fundamental equations, one can derive all the related parameters of a simple pendulum. Note that the above equations holds good for small-angle approximation.

For amplitudes beyond the small angle approximation,

The time-period is given by:

$$
T=4 \sqrt{\frac{l}{2 g}} \int_{0}^{\theta_{0}} \frac{1}{\sqrt{\cos \theta-\cos \theta_{0}}} d \theta
$$

Note that this integral diverges as $\theta_{0}$ approaches the vertical:

$$
\lim _{\theta_{0} \rightarrow \pi} T=\infty
$$

so that a pendulum with just the right energy to go vertical will never actually get there. Conversely, a pendulum close to its maximum can take an infinitely long time to fall down.

\section{Comparison of four different approaches used in this paper:}

This paper used four different approaches to calculate the responses of the pendulum [2]:

Euler method: Consider the case of simple pendulum with length I, in restoring force of gravity with acceleration g. In the approximation that the pendulum is not driven with large angle $\theta$, the equation of motion is given by (1).

$$
\frac{d^{2} \theta}{d t^{2}}=-\frac{g}{l} \theta
$$

The analytical solution to this differential equation is given by:

$$
\begin{aligned}
& \theta=\theta_{0} \sin (\Omega t+\Psi) \\
& \text { Where, } \Omega=\sqrt{\frac{g}{l}}
\end{aligned}
$$

Euler approximation is based on approximation of two first order differential equations:

$$
\begin{aligned}
& \frac{d \omega}{d t}=-\frac{g}{l} \theta \\
& \frac{d \theta}{d t}=\omega
\end{aligned}
$$

From the definition of derivative, we can state that:

$$
\omega(t+\Delta t)=\omega(t)-\frac{g}{l} \theta(t) \Delta t
$$


Likewise, for $\theta$

$$
\theta(t+\Delta t)=\theta(t)+\omega(t) \Delta t
$$

This approximation to the solution of differential equations is known as Euler Method.

Euler-Cromer method: The results of Euler method are unstable and violate physics since the approximation is valid for only small-angles. A stable approach was given by Cromer based upon the advanced $\omega$ to calculate the advanced $\theta$, i.e, use $\omega_{i+1}$ to calculate $\theta_{i+1}$. With this minor change, the numerical solution becomes stable and expected results are obtained.

$2^{\text {nd }}$ order Runge-kutta method: This method gives the most stable and expected results same as the EulerCromer method. Given a vector $\phi_{n}$ of unknowns at time $t_{n}$, and the first order differential equation

$$
\frac{d \phi}{d t}=f(\phi, t)
$$

The second order Runge-Kutta estimate for $\phi_{n+1}$ is given by:

$$
\begin{gathered}
k_{1}=\Delta t \cdot f\left(\phi_{n}, t_{n}\right) \\
k_{2}=\Delta t \cdot f\left(\phi_{n}+\frac{k_{1}}{2}, t_{n}+\frac{\Delta t}{2}\right) \\
k_{3}=\phi_{n}+k_{2} \\
\text { Where, } \Delta t=t_{n+1}-t_{n}
\end{gathered}
$$

Since the method is explicit; equation (12) doesn't require a non-linear solver if the function is non-linear.

ODE-23 MATLAB® solver: The solution using the built in MATLAB® solver ODE23 is somewhat less straightforward than those using the other techniques. MATLAB® has a very specific way to define a differential equation, as a function that takes one vector of variables in the differential equation, plus a time vector, as an argument and returns the derivative of that vector. The only way that MATLAB ${ }^{\circledR}$ keeps track of which variable is inside which vector is the order the user choose to use the variables in. one can define the differential equations based on that ordering of variables in the vector, define the initial conditions in the same order, and the columns of the results will also be in that order.

The equations governing the motion of simple and damped, non-linear pendulum are simulated and evaluated using these four approaches in MATLAB®.

The simulation results and the comparison of the results from these methods is provided in section 2.1 and 2.2 respectively.

\section{SIMULATION RESULTS:}

The solution of the four methods as described in the previous sections was simulated in MATLABß [8]. This is because MATLAB is a very powerful tool computing system for handling the calculations involved in scientific and engineering problems.

With MATLAB, computational and graphical tools to solve relatively complex science and engineering problems can be designed, developed and implemented.

In this section, the simulation results are presented. For modelling of the pendulum, a range of time is selected and the aforementioned equations are used for plotting the results in Matlab. The results are provided for two scenarios:

a) The angle $\theta$ traversed by the pendulum in a given time.

b) The measured displacement of the pendulum over a given span of time.

The results are presented for both simple and physical pendulums to clarify the difference in operation. 


\section{Solution using the Euler method:}

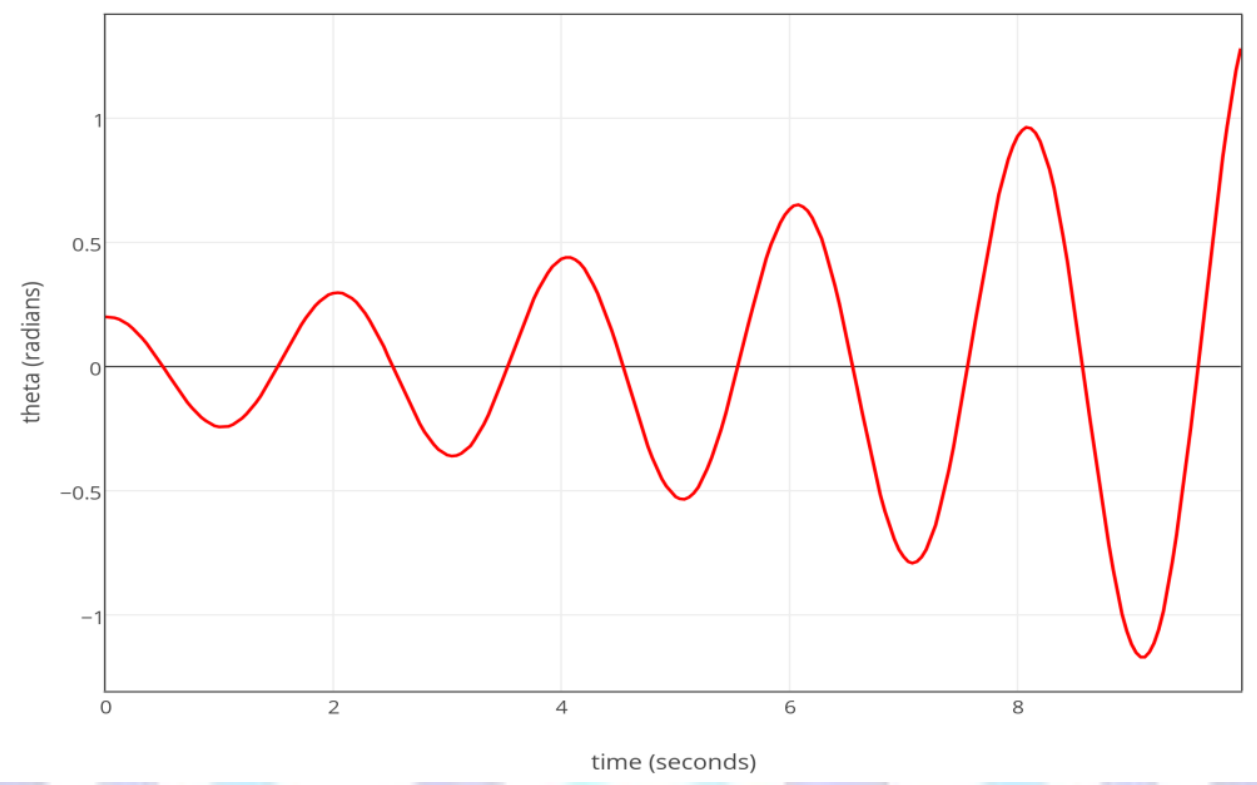

Figure 1: Euler Method

Note that the oscillations grow with time. Hence, this approach is unstable.

\section{Solution using the Euler-Cromer method:}

This problem with growing oscillations is addressed by performing the solution using the Euler - Cromer method. The result is shown below:

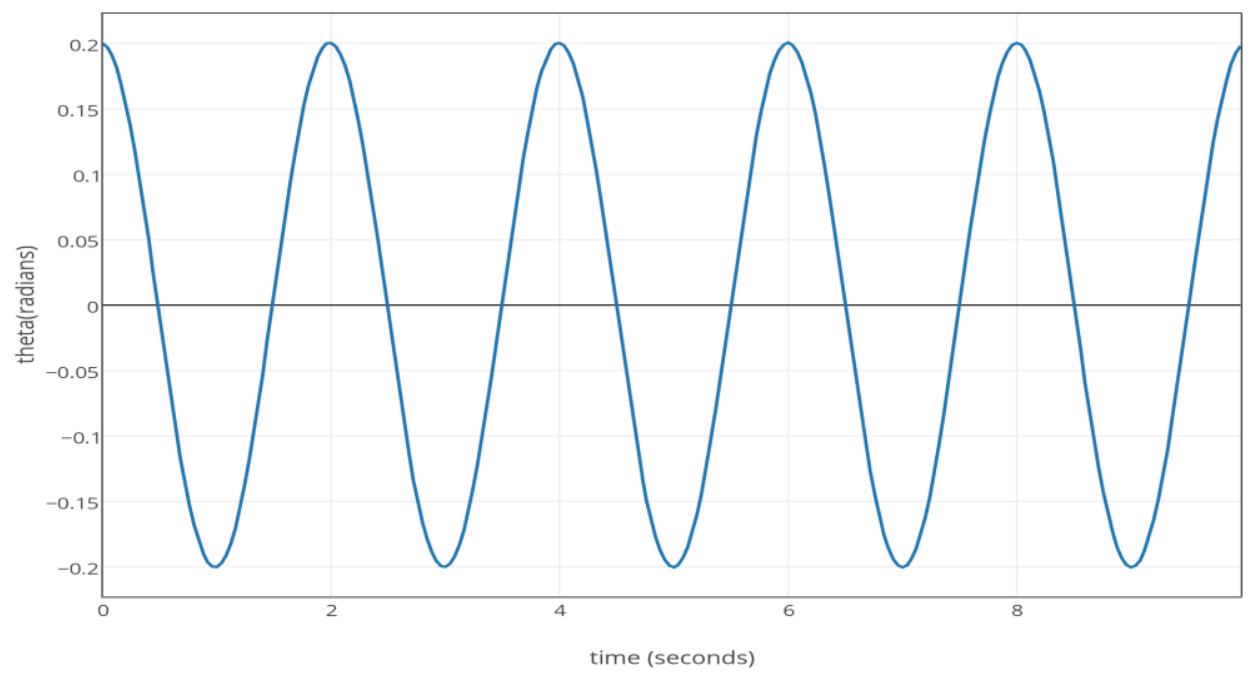

Figure 2: Euler-Cromer Method

The result proves that Euler-Cromer method is stable. 


\section{Solution using the Runge-Kutta Method:}

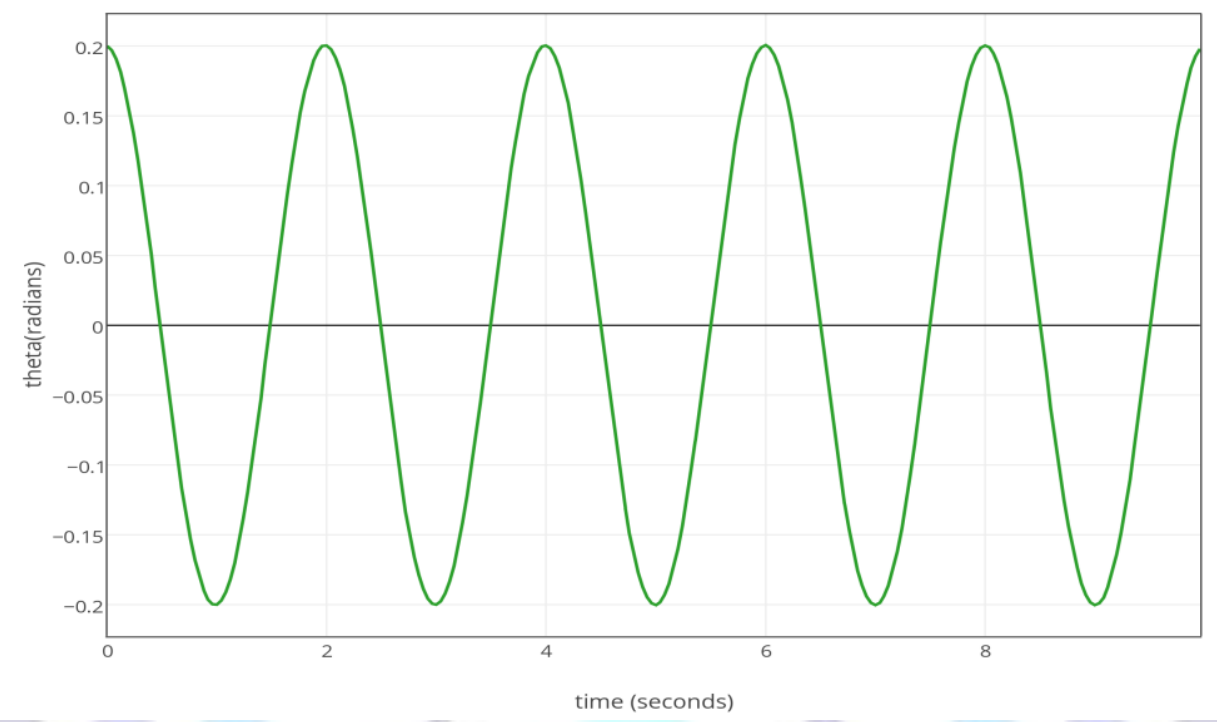

Figure 3: Runge-Kutta Method

As can be easily witnessed, this method yields identical results as the Euler-Cromer method. This is probably because both the methods are very similar in operation.

\section{Solution using the ODE-45 MATLAB solver:}

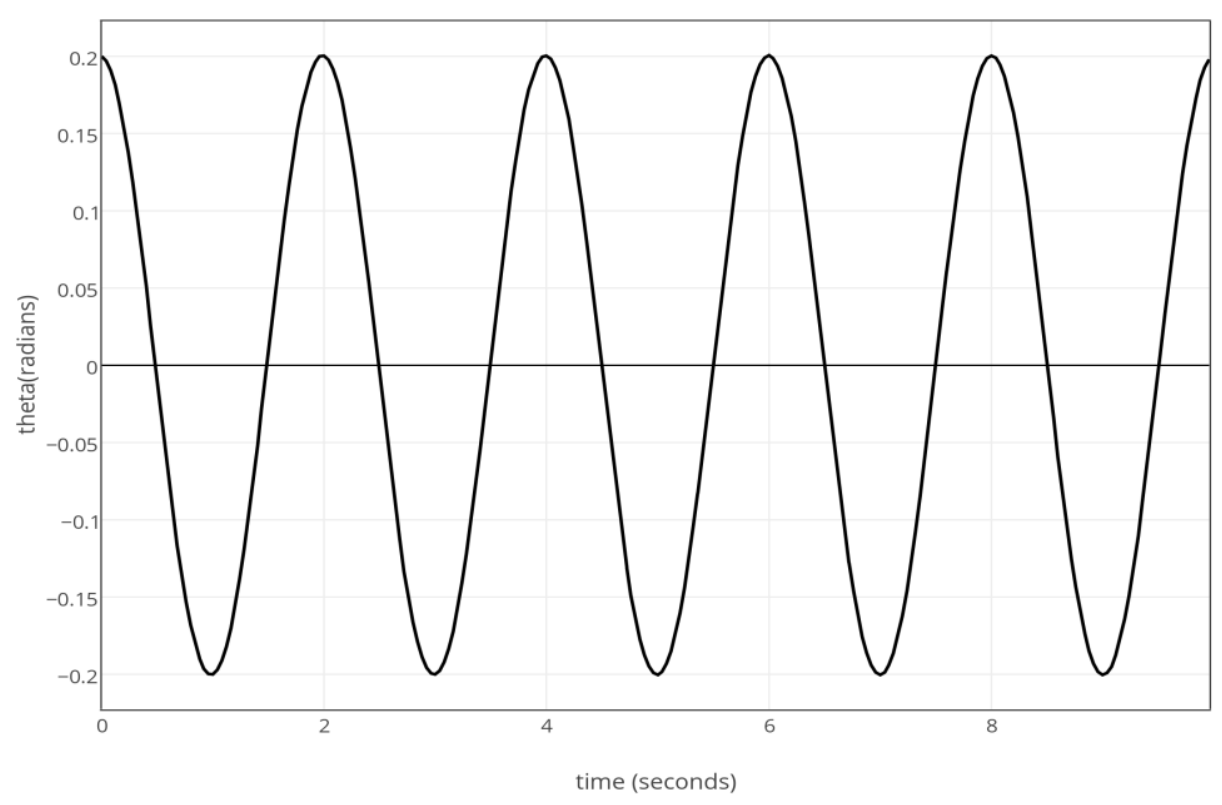

Figure 4: ODE-45 Solver

This approach is based on Runge-Kutta method and is an inbuilt MATLAB solver. The results prove the point that both these approaches are identical in operation. 


\section{COMPARISON OF DIFFERENT APPROACHES:}

In this section, the performance of the aforementioned approaches are compared. The displacement calculation was carried out using the equations presented in the preceding sections and the results are plotted in Matlab.
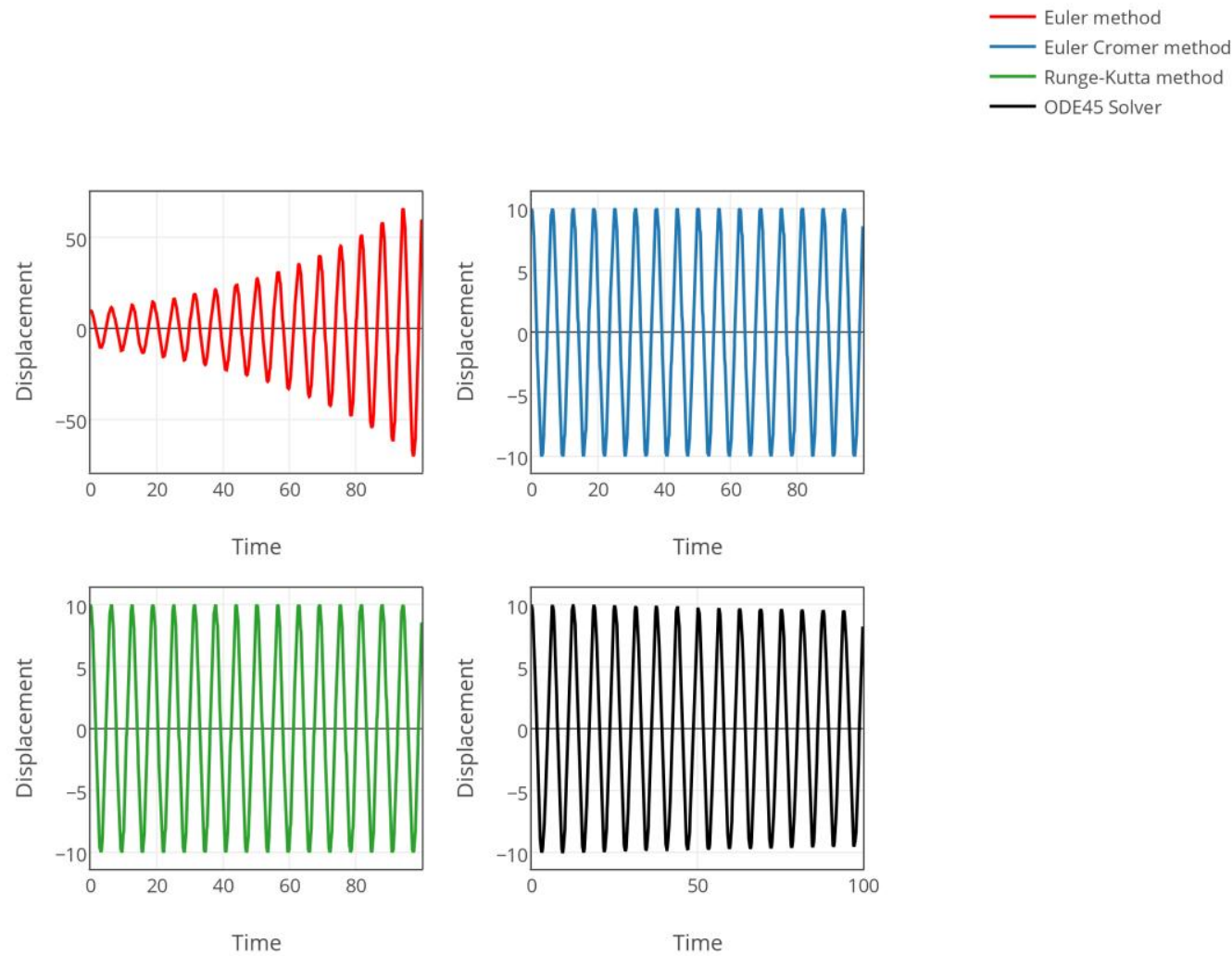

Figure 5: Comparison of different approaches

By visual inspection of the plots, one can establish the fact that displacement is directly correlated with the angle $\theta$. It can be intuitively understood from the motion of the pendulum. The more $\theta$ traversed by the pendulum, the larger is the displacement from the initial state. The results also present the fact that except the Euler-method, all the other approaches provide a stable approach and the responses are identical in magnitude and shape. These results are simulated for a simple ideal pendulum where there is no friction taken into account. However, in reality, the damped or non-linear pendulum is a good fit as it takes friction into account.

Next, the paper presents the results for a damped, non-linear pendulum using the Euler-Cromer approach. The results from Runge-kutta and ODE-23 solver will also be identical to the Cromer approach as presented in the previous sections.

The motivation for choosing the Euler-Cromer approach arises from the following merits:

1. This approach is stable as proved in the preceding sections.

2. The implementation is simple.

3. Physical modelling of the pendulum is robust as this approach is based on physical laws governing the motion of the pendulum.

4. This method takes into account the dynamic nature of the pendulum and adapts to the changes. 


\section{Solution for a damped pendulum using the Euler-Cromer method:}

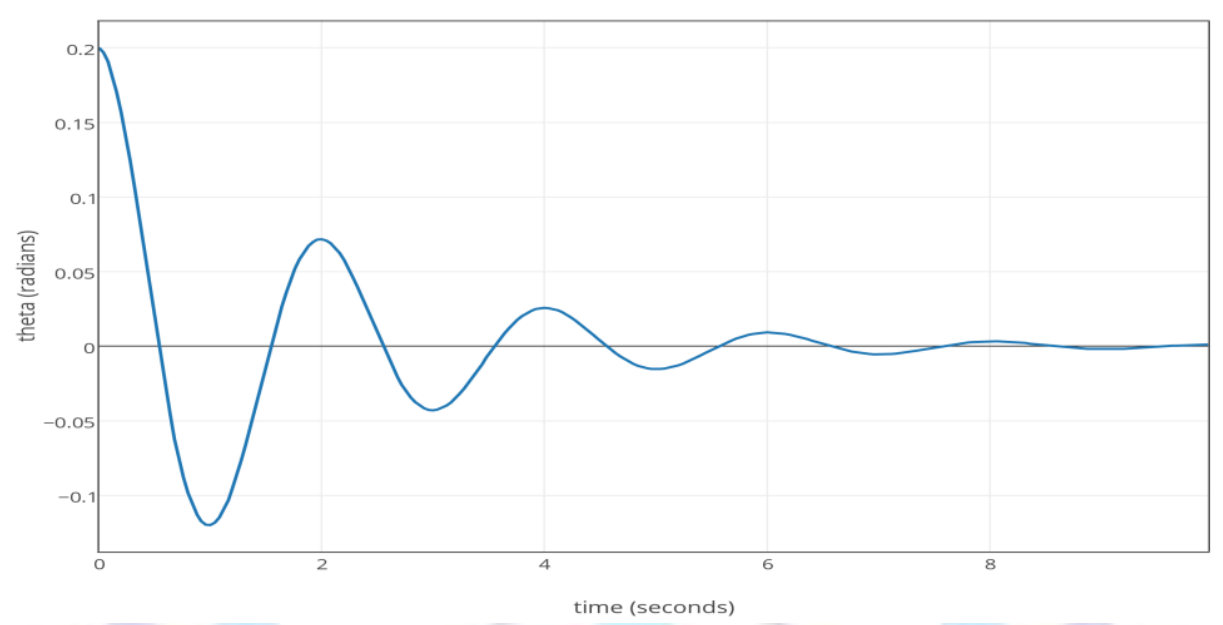

Figure 6: Damped pendulum using Euler-Cromer Approach

This figure tells us that in damping pendulum, the oscillations decrease or slow down with time. This happens because of the friction in the surface and air-drag. Hence, in comparison to simple pendulum, the damped pendulum's amplitude decreases with time.

The paper next analyses the non-linear damped and driven pendulum also known as the physical pendulum. In this type of pendulum, the oscillations become stable instead of decaying with time. The reason behind this behavior is damping strength. The damping strength limits the decay of the pendulum to a specific period. After that period, the pendulum behaves as an ideal pendulum with stable oscillations.

In this sub-section, the characteristics of the physical pendulum using different values of damping strength is analyzed in MATLAB®. The results were shown by applying Euler-Cromer method.

Solution for a non-linear, damped, driven pendulum: Physical pendulum, using the Euler-Cromer method:

\section{Damping Strength $=0.5$}

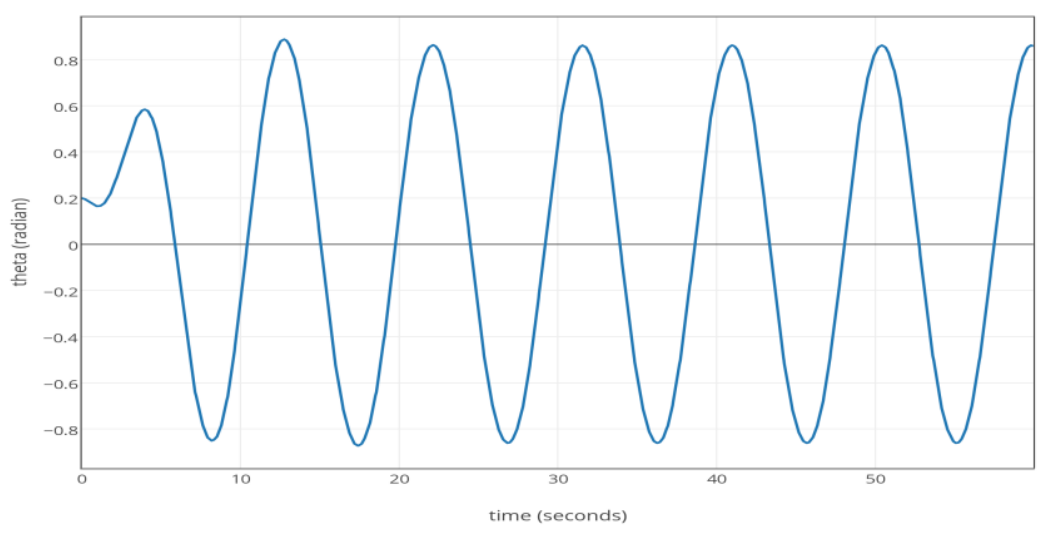

Figure 7: Damped-Driving pendulum using damping strength $=0.5$ 
As clearly witnessed from the figure, the physical pendulum behaves like a simple pendulum if damping strength is small. As the damping strength increases, the physical pendulum diverges from the simple pendulum and gives irregular patterns as shown in the next figure. In real life scenarios, the damping strength plays a critical role as it is impossible to provide in advance. The strength depends on factors such as friction, noise, external disturbances etc.

\section{Damping Strength $=1.2$}

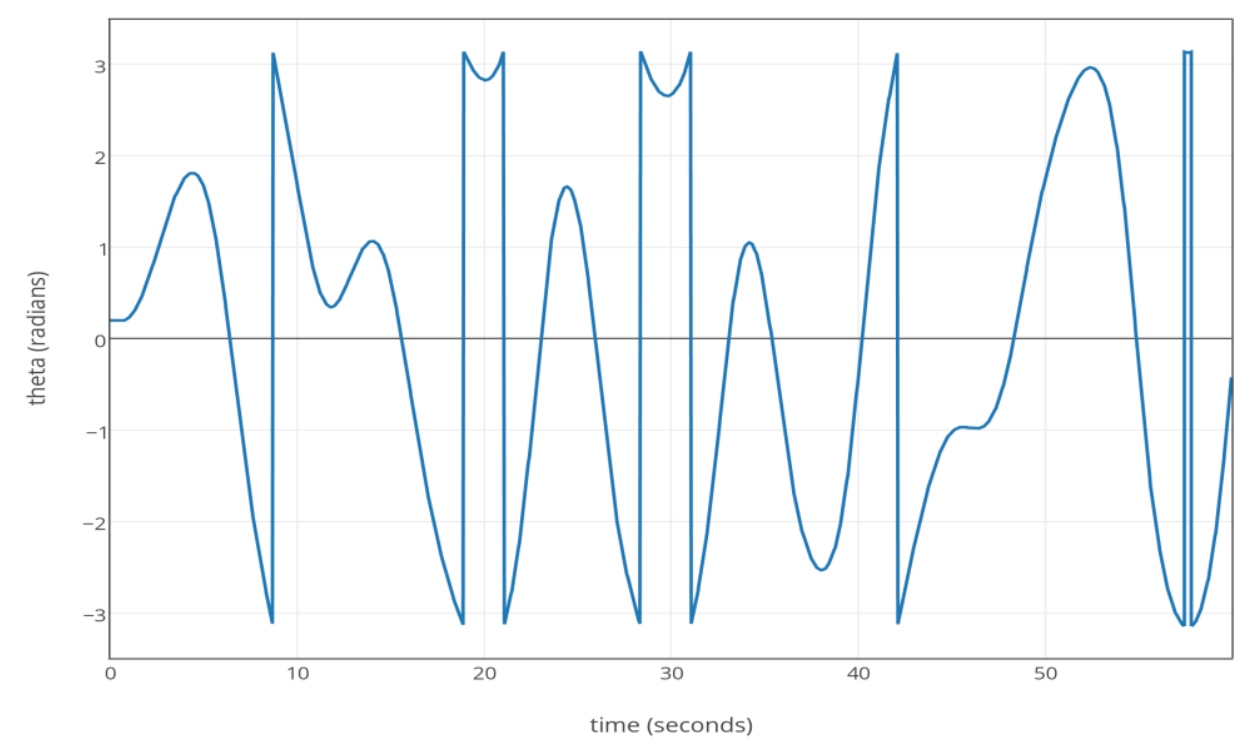

Figure 8: Damped Driving Pendulum using damping strength = 1.2

The next section presents the experimental setup for validating the simulations carried out in the preceding sections. The experiments were carried out in Radar Lab as a courtesy of Electrical and Computer Engineering Department of Mississippi State University.

\section{EXPERIMENTAL SETUP:}

In this section, the paper talks about the radar returns of a simple pendulum and discussion on micro-doppler effects asscociated with it. The radar used for the experiments was originally designed and developed by lincoln labaratory, MIT. The design and fabrication of the RADAR is available as an open-source[3]. The idea is modified to design a CANTENNA RADAR which operates in three different modes: Doppler Mode, Synthetic Aperture Mode and Ranging Mode. For the purpose of this paper, the RADAR was operated in Doppler mode for calculating the micro-doppler signatures associated with the pendulum.

\section{Micro-doppler effects of simple pendulum:}

To calculate radar backscattering from an oscillating pendulum, ordinary differential equations given in section I and II are used for solving the swinging angle and the angular velocity. Therefore, at each time instant during a radar observation time interval, the location of the pendulum can be determined. Based on the location and orientation of the pendulum, the RCS of the pendulum and the radar received signal can be calculated[4]. In this section, different parameters are evaluated for the experiment and a brief study of the radar is performed.

\section{RADAR Hardware:}

The RADAR used in this paper can work in three different modes: doppler mode, ranging mode, and synthetic imaging mode. However, for studying the micro-doppler effects, we used the RADAR in doppler-mode. 
Table 1: NOTIONAL RADAR PARAMETERS

\begin{tabular}{|c|c|}
\hline Mode of operation & FMCW \\
\hline Frequency-range & ISM band of 2.4Ghz \\
\hline Transmitted power & $10 \mathrm{~mW}$ \\
\hline Maximum Range & $1 \mathrm{~km}$ for $10 \mathrm{dBsm}$ targets \\
\hline
\end{tabular}

\section{Fully assembled RADAR kit:}

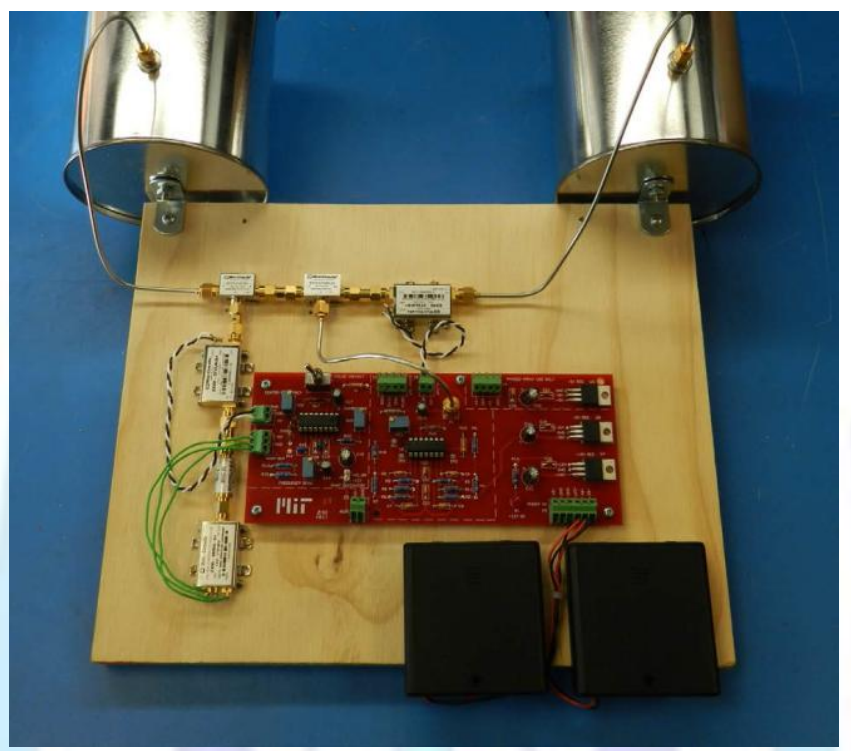

Figure 9: RADAR setup (Courtesy: Radar LAB, MSU)

\section{Block-diagram:}

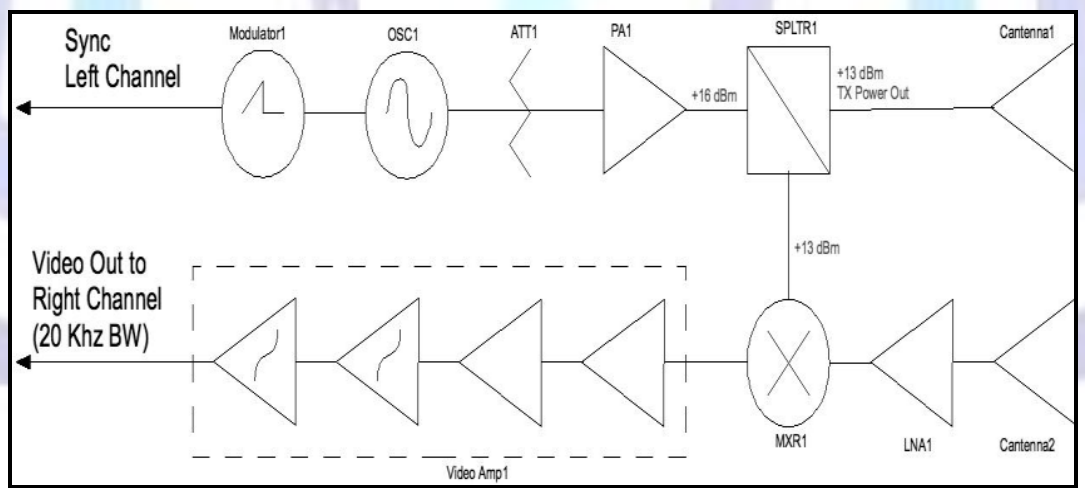

Figure 10: Schematic Block Diagram

Table 2 : Experimental Parameters

\begin{tabular}{|l|l|}
\hline Speed of light & $2.99 \times 10^{6} \mathrm{~m} / \mathrm{s}$ \\
\hline Coherent processing interval & 0.50 \\
\hline Center frequency & $2.590 \times 10^{6} \mathrm{~Hz}$ \\
\hline Length of pendulum & $10^{-3} \mathrm{~m}$ \\
\hline No. of oscillations & 10 \\
\hline Maximum speed of oscillations & $10 \mathrm{~m} / \mathrm{s}$ \\
\hline RADAR range from the pendulum & $10 \mathrm{~m}$ \\
\hline
\end{tabular}


In the next section, the experimental results are presented. The CANTENNA RADAR was operated in the Doppler mode for recording the Doppler signatures of the pendulum. The RADAR was operated in a closed environment to reduce the effect of noise on the results.

\section{EXPERIMENTAL RESULTS:}

The RADAR was operated in doppler mode to record the doppler returns from the pendulum. It was kept at a distance of $10 \mathrm{~m}$ from the pendulum and the pendulum was oscillated 10 times back and forth. The observations were recorded in audio-format (.WAV file) and it was processed and analyzed in MATLAB. The results from the experiment shows the micro-doppler effects of a simple pendulum recorded by the RADAR.

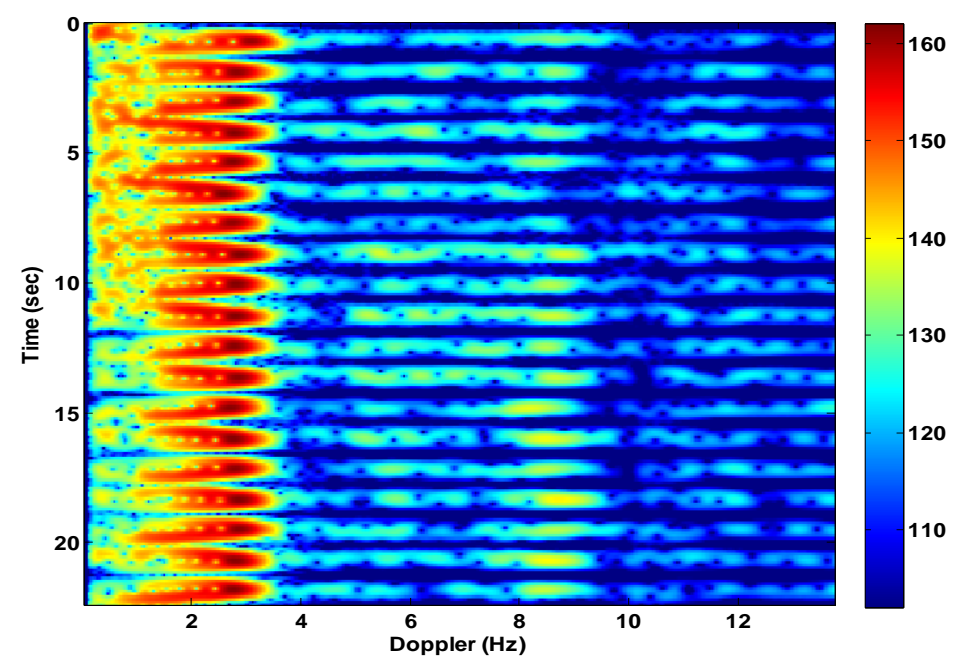

Figure 11: Experimental Results

As clearly evident from the results, the micro-doppler returns from the RADAR clearly verifies the theoretical results of the simple pendulum modeled in section 2. The precise RADAR returns from the simple pendulum also proves the proper functioning of the RADAR in Doppler Mode, signifying its efficiency. The blue lines shown in the figure are a result of noise/disturbance caused by human motion behind the target.

The experimental results are in direct coherence with the analytical results discussed in the preceding sections. This agreement validates the physical modelling of the pendulum and proper operation of the CANTENNA RADAR. The Doppler signatures are directly related to the physical laws governing the motion of the pendulum and the experimental results also validate this fact. The motivation for selecting simple pendulum as the test target arises from the fact that pendulum is a widely discussed object in physics and the laws governing its motion are well-establsihed.

\section{FUTURE WORK:}

In the future work, all the parameters of the pendulum and all the modes of the pendulum will be modeled using the RADAR. The operation of the RADAR will also be tested in all the three modes to validate the experiments. This paper attempts to provide a strong frame-work for studying doppler phenomenon associated with moving targets. Our next goa would be to study the micro-doppler effects of humans, moving vehicles etc. and to calculate additional parameters of the pendulum. We will also implement noise-cancellation features in the RADAR to make it more robust.

\section{ACKNOWLEDGEMENT:}

I would sincerely like to thank Dr. John Ball who have guided and participated in the discussions related to the paper. I would also like to extend my sincere thanks to my colleagues and students who shared their thoughts and ideas leading to the completion of this paper.

\section{CONCLUSION:}

In this paper, the micro-doppler signatures associated with a simple pendulum is studied. The physical modelling of the pendulum was carried out based on the equations governing the motion, velocity and angular accelaeration. In the first section, a brief introduction of pendulum is provided. Second section presented the fundamental differential equations and subsequently the modelling of the pendulum using four different approaches were carried out in Matlab. The theoretical results are provided for all the three modes of pendulum. In section 4 , the experimental setup involving the cantenna radar 
was demonstrated. Finally, the experimental results were implemented using a cantenna radar employing simple pendulum as the test target. The results show a strict correlation with the theoretical modelling and simulations.

\section{REFERENCES:}

I Michael R. Matthews, Colin F. Gauld and Arthur Stinner, The Pendulum Springer, 2005.

II N.Giordano and H.Nakanishi, Computational Physics, Prentice Hall, 2006.

III MIT open course-ware, http://ocw.mit.edu/.

IV Victor C. Chen, The Micro-Doppler Effects inRadar, Artech House, 2011. 